\title{
Stress Fracture of Bilateral Distal Tibia Provoked by Stair Climbing
}

\author{
Aydin Arslan ${ }^{*}$, Ali Utkan ${ }^{2}$, Tuba Tulay Koca ${ }^{3}$ \\ ${ }^{1}$ Department of Orthopedics, State Hospital, Malatya, Turkey \\ ${ }^{2}$ Department of Orthopedics, Ankara Numune Training and Research Hospital, Ankara, Turkey \\ ${ }^{3}$ Department of Physical Therapy and Rehabilitation, State Hospital, Malatya, Turkey \\ Email: draarslan@hotmail.com
}

Received 22 September 2015; accepted 19 October 2015; published 22 October 2015

Copyright (C) 2015 by authors and Scientific Research Publishing Inc.

This work is licensed under the Creative Commons Attribution International License (CC BY). http://creativecommons.org/licenses/by/4.0/

(c) (i) Open Access

\begin{abstract}
Background: Stress fractures are fatigue-induced, very small cracks in the bone caused by repetitive application of force and most often seen in the weight-bearing bones of the lower extremities of athletes or military recruits. Case Report: A 47-year-old postmenopausal and heavy smoker woman complained of localized sensitivity on the distal-anterior and medial surfaces of both tibiae, which had been ongoing for 1 month. She moved to a flat at 5 th-floor without an elevator a year ago. Bilateral stress fractures were diagnosed by radiographs and confirmed by Magnetic Resonance Imaging (MRI). She was suggested to restrict activities and to use crutches until the symptoms subsided. Bilateral ankle joint pneumatic brace with full heel support was applied. The patient was followed one year after diagnosis. At the 2-month follow-up examination, the patient was pain free and planning to move to a new house. Conclusions: Several risk factors have been defined in the pathogenesis of stress fracture. In the present case, the acute phase had passed and diagnosis could be made from the direct radiographs. Although extremely rarely seen, tibia distal stress fracture should be kept in mind in the differential diagnosis of patients with the relevant risk factors who present with tibia distal localized pain.
\end{abstract}

\section{Keywords}

Stress Fracture, Tibia, Tibia Distal

\section{Introduction}

A stress fracture is defined as fatigue-induced very small cracks in the bone caused by repetitive application of

${ }^{*}$ Corresponding author. 
force such as running or jumping and is common in athletes and military recruits. Although stress fractures are seen throughout the body, they are generally more often seen in the weight-bearing bones of the lower extremities, the tibia, tarsal and metatarsal bones [1]-[3].

In this paper, the diagnosis and treatment process were examined of the case of a 47 -year-old female who presented with a 1-month history of progressive pain in the distal and medial of both tibiae. Informed consent had been obtained from the patient.

\section{Case}

A 47-year-old female patient presented with a 1-month history of localized sensitivity in both tibia distal anterior and medial surfaces. The pain was described as present at rest and significantly increasing on weightbearing. With palpation, mild edema and excessive pain were determined in both tibiae in the anterior and medial surfaces towards the ankle. On direct radiographs periosteal new bone formation on the medial cortex and sclerotic areas in the distal metaphysis were determined (Figure 1). On the T1-weighted coronal magnetic resonanance image a linear areas of low signal intensity in the bilateral distal tibia were seen (Figure 2).

The patient had no chronic disease and entered menopause 7 years previously. The patient had moved to a $5^{\text {th }}$-floor home in an apartment block without an elevator one year previously and she smoked cigarettes 20 pack-year. Blood tests were performed. Blood electrolytes, kidney and liver function tests, sedimentation rate, C-reactive protein, FSH, LH, thyroid function tests, parathormone levels were normal. Vitamin D level was 8.31 $\mathrm{ng} / \mathrm{mL}$ (10 - 44 is normal level). Lumbar vertebrae bone mineral density measurements were applied. Total
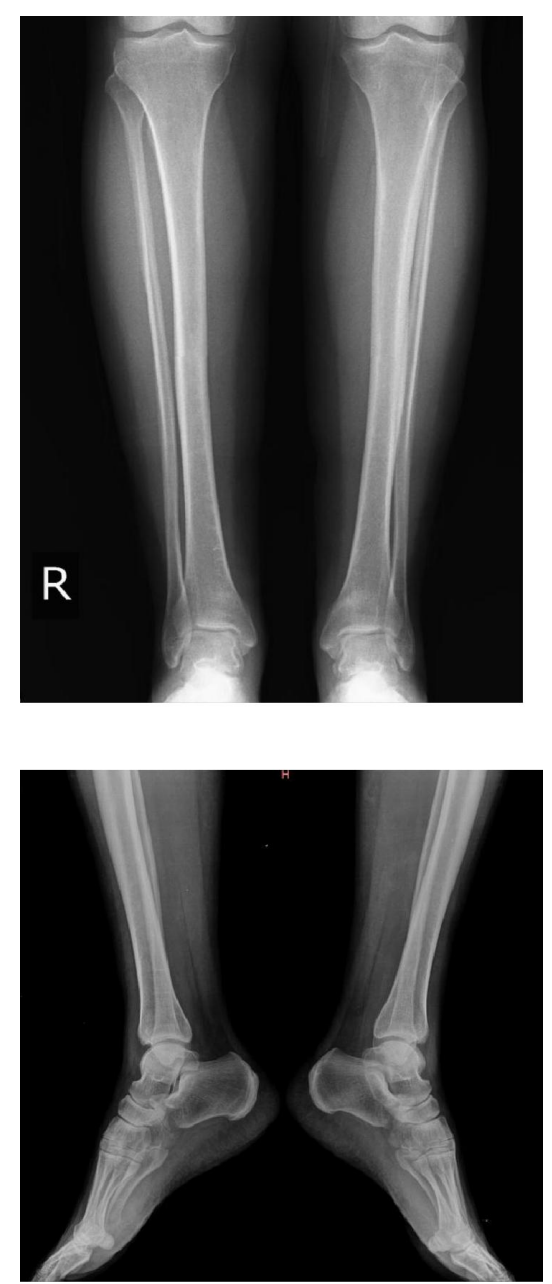

Figure 1. Antero-posterior and lateral plain radiographs of tibia and ankle. 


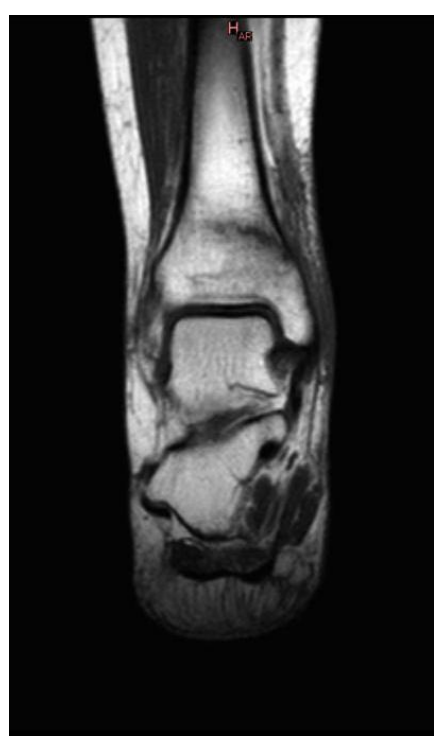

right ankle



left ankle

Figure 2. T1 weighted coronal magnetic resonance image of bilateral distal tibia and ankle.

lumbar $\mathrm{T}$ and $\mathrm{Z}$ scores were -1.5 and -1.1 respectively. A double pneumatic compression brace (air cast ankle brace) with full heel support was recommended. Flurbiprofene $100 \mathrm{mg}$ orally twice daily (Sanovel, Istanbul, Turkey) and Calcium 1200 mg with 800 IU vitamin D twice daily (Mentis, Istanbul, Turkey) were given to the patient. Restriction of activity was implemented and the patient was permitted to be mobile with crutches for critical needs. The patient was followed one year after diagnosis.

At the follow-up examination 1 month later, the complaints of pain decreased. At the 2-month follow-up, the pain recovered and the patient returned to normal activities. The patient was recommended to move to a more suitable house and to stop smoking.

\section{Discussion}

Stress fractures are most often seen in the tibia. Although there is no consensus, as the pathogenesis of stress fracture is multifactorial, several risk factors have been identified. Some of these risk factors are advanced age, female gender, white ethnicity, vitamin D receptor polymorphism, low vitamin D levels, high levels of parathormone, low bone mineral density and content, tall height, poor general condition, iron deficiency, steroid use, bisphosphonate treatment, cigarette smoking, alcoholism [2] [4] [5]. With repeated overloading, because the increase in osteoclastic activity is greater than osteoblastic activity in the stress fracture process, microfractures and defects in the trabecular structure develop as this newly-formed bone tissue is weaker and more porotic and with progression of the disease, callus formation develops around these microfractures [5].

In the differential diagnosis of tibia distal stress fracture, medial tibial stress syndrome should be taken into consideration. In medial tibial stress syndrome, pain is felt more widely in the mid-distal and posteromedial of the tibia. In tibia distal stress fracture, as in the current patient, the pain is more localized. In addition, in medial tibial stress syndrome, edema does not develop in the cruris, whereas in a stress fracture, edema is seen [6]. Malignant tumours must be taken into account in the differential diagnosis [3]. In contrast to stress fractures, pathological fractures occur under normal stress as a result of weakened bone structure for reasons such as tumour, infection or osteoporosis [4].

In diagnosis, firstly, plain radiographs should be taken. Bone edema as an early finding of stress fracture cannot be seen on plain radiographs so acute stress fracture may not be able to be diagnosed. The formation of a fracture line, cortical irregularity and periosteal new bone formation become evident after 3 weeks [2] [7]. In the current case, as the acute phase had passed, diagnosis could be made from the periosteal callus formation and the sclerotic areas on the plain radiograph. Magnetic resonance imaging is the gold standard in diagnosis and has the advantage of being able to show edema in the soft tissue and bone [8]. Computed tomography is useful in the 
differentiation of bone malignancies. However, the disadvantages are the exposure to radiation and it does not show soft tissue. Although scintigraphy has high sensitivity, the fracture line cannot be seen directly and exposure to radiation are disadvantages. Furthermore, scintigraphy may give a false positive result when there is local infection or a tumour. The use of ultrasound is limited [7] [9].

Stress fractures are classified as low-risk or high-risk. As low-risk fractures are compression stress fractures, they can recover with conservative treatment. They are generally seen in the femoral shaft, the medial tibia, the fibula, calcaneus and first and fourth metatarsals. As the high-risk stress fractures are tension-typed , surgical intervention is generally required. Delayed union and non-union may be seen in this type of fracture. They are generally seen in the $5^{\text {th }}$ metatarsal, the anterior tibia, the tarsal navicular bone, the femoral neck, the patella and the $1^{\text {st }}$ metatarsal sesamoid bone [1] [8] [9]. According to this classification, the current patient should be evaluated as a low-risk stress fracture.

In the treatment, it is necessary to reduce activity from as soon as diagnosis is made until the complaints of pain is completely recovered. This could take 3 months or longer [2]. Anti-inflammatory-analgesic drugs can be used to reduce the pain [2]. Since osteopenia and low vitamin D level were found as risk factors in our patient, we gave the patient a calcium vitamin $\mathrm{D}$ tablet twice daily. The use of a pneumatic compression brace in tibia stress fractures has been reported to shorten the period of restricted activity and decrease pain [10]. In the current case, a pneumatic compression brace was applied. In a previous study conducted on soldiers, the use of shock-absorbing orthoses in the shoes was shown to reduce the risk of stress fractures developing [10].

\section{Conclusion}

Stress fractures are seen most frequently in the tibia and the pathogenesis is multi-factorial. In the current case, the stress fracture occurred due to a sudden sharp increase in activity with ascending five flights of stairs to the home. Cigarette smoking, low vitamin D level and osteopenia were identified as risk factors. When the patient presented, the acute phase of the stress fracture had passed and periosteal new bone formation was observed on the direct radiograph. As the disease is generally seen as normal on direct radiographs for the first $2-3$ weeks, there is a possibility of delayed diagnosis. Although extremely rarely seen, tibia distal stress fracture should be kept in mind in the differential diagnosis of patients with associated risk factors who present with tibia distal localized pain.

\section{Acknowledgements}

No funding sources.

\section{References}

[1] Boden, B.P., Osbahr, D.C. and Jimenez, C. (2001) Low Risk Stress Fractures. American Journal of Sports Medicine, 29, 100-111.

[2] Patel, D.S., Roth, M. and Kapil, N. (2011) Stress Fractures: Diagnosis, Treatment, and Prevention. American Family Physician, 83, 39-46.

[3] Fayad, L.M., Kamel, I.R., Kawamoto, S., Bluemke, D.A., Frassica, F.J. and Fishman, E.K. (2005) Distinguishing Stress Fractures from Pathologic Fractures: A Multimodality Approach. Skeletal Radiology, 34, 245-259. http://dx.doi.org/10.1007/s00256-004-0872-9

[4] Iwamoto, J., Sato, Y., Takeda, T. and Matsumoto, H. (2011) Analysis of Stress Fractures in Athletes Based on Our Clinical Experience. World Journal of Orthopaedics, 2, 7-12. http://dx.doi.org/10.5312/wjo.v2.i1.7

[5] Scaglione, M., Fabbri, L., Dell’Omo, D., Gambini, F., Di Rollo, F. and Guido, G. (2014) A Case of Bilateral Stress Fractures in an Old Woman: Three Years of Pain. Clinical Cases in Mineral and Bone Metabolism, 11, 149-152. http://dx.doi.org/10.11138/ccmbm/2014.11.2.149

[6] Batt, M.E., Ugalde, V., Anderson, M.W. and Shelton, D.K. (1998) A Prospective Controlled Study of Diagnostic Imaging for Acute Shin Splints. Medicine \& Science in Sports \& Exercise, 30, 1564-1571. http://dx.doi.org/10.1097/00005768-199811000-00002

[7] Spitz, D.J. and Newberg, A.H. (2002) Imaging of Stress Fractures in the Athlete. Radiologic Clinics of North America, 40, 313-331. http://dx.doi.org/10.1016/S0033-8389(02)00010-6

[8] Boden, B.P. and Osbahr, D.C. (2000) High Risk Stress Fractures: Evaluation and Treatment. Journal of the American Academy of Orthopaedic Surgeons, 8, 344-353. 
[9] Kahanov, L., Eberman, L.E., Games, K.E. and Wasik, M. (2015) Diagnosis, Treatment, and Rehabilitation of Stress Fractures in the Lower Extremity in Runners. Open Access Journal of Sports Medicine, 6, 87-95. http://dx.doi.org/10.2147/OAJSM.S39512

[10] Rome, K., Handoll, H.H. and Ashford, R. (2005) Interventions for Preventing and Treating Stress Fractures and Stress Reactions of Bone of the Lower Limbs in Young Adults. Cochrane Database of Systematic Reviews, 2, Article ID: CD000450. http://dx.doi.org/10.1002/14651858.cd000450.pub2 